\title{
Quantitative Evaluation of Diabetic Foot Wound Healing Using Hydrogel Composite Nanosilver (AgNPs) - Based Dressing Vs. Traditional Dressing: A Prospective Randomized Control Study
}

\author{
EHSAN A. YAHIA ${ }^{1}$, AYMAN E. EL-SHARKAWEY², MAGDA M. BAYOUMI ${ }^{3,4}$ \\ ${ }^{1}$ Medical-Surgical Nursing Department, Faculty of Nursing, Cairo University \\ ${ }^{2}$ Nanoscopic center, Kuwait University. \\ ${ }^{3}$ Medical-Surgical Nursing Department, Faculty of Nursing, Beni-Suef University. \\ ${ }^{4}$ Nursing Department, College of Health and Sport Sciences, University of Bahrain. \\ (ClinicalTrials.gov Identifier: NCT04834245).
}

\begin{abstract}
Background: The wound dressings perform a crucial role in cutaneous wounds management due to their ability to protect wounds and promote dermal and epidermal tissue regeneration.

Aim: the aim was to evaluate the effectiveness of using hydrogel/nano silver-based dressing vs. traditional dressing on diabetic foot wound healing.

Methods: Sixty patients with type-2 diabetes hospitalized for diabetic foot wound treatment were recruited from selected Surgical departments. A prospective randomized control study was carried, and the results showed that the percentage of a reduction rate of the ulcer by the third week of the treatment as in the hydrogel/nano silverbased dressing group was higher (15.11\%) than traditional wound dressing group (33.44\%). Moreover, the mean ulcers size "sq mm" in the hydrogel/nano silver-based dressing group recognized a faster healing rate (15.11 \pm $7.89)$, and considerably lesser in comparison to the traditional by the third week (21.65 \pm 8.4$)$.

Conclusion: The hydrogel/nanosilver-based dressing showed better results than traditional dressing in managing diabetic ulcer foot.
\end{abstract}

Keywords: Diabetes, Wound, Diabetic ulcer, Wound dressing, Nanomedicine, Nano-silver.

\section{INTRODUCTION}

Diabetes is a group of metabolic diseases characterized by a state of an increased level of glucose in the blood related to defects in insulin section and/ or action which lead to acute and long-term complications (ADA, $(1,2)$. One of the most severe diabetic complications resulting in substantial mortality and morbidity is Diabetic Foot Ulcers (DFUs). The associated mortality rate with the DFU development is estimated to be $5 \%$ in the initial 12 months and $42 \%$ in the 5-years ${ }^{(3)}$.

Unfortunately, there is an elevation in the incidence of diabetes worldwide, particularly in the Arab world. Noteworthy, in developing countries, the Diabetic Foot Ulcer (DFU) has been estimated at $15 \%$ and recognized as the most common complication of diabetes which leading cause for disability, morbidity, and morbidity ${ }^{(4)}$.

In Egypt, the prevalence of diabetic foot ulcers is high. The frequently stated reasons for this prevalence include lack of knowledge regarding diabetic foot problems, foot care measures, and inappropriate footwear. Those reasons are pertinent to Egyptian patients since more than $90 \%$ of the people with diabetes have a lack of knowledge about the disease process and the complications as diabetic foot problems and its care measures ${ }^{(5)}$. Subsequently, there is vast of early morbidity and mortality associated with diabetes is documented, hence the prompt diagnosis of foot problems and early intervention can avert many amputations $^{(6)}$.

Indeed, the disease's burden is rising and the DFU in people with diabetes has a significant financial impact through primary care, community care, outpatient costs, increased bed occupancy, and prolonged stays in hospital (7). Interferences for reducing the diabetic foot amputation and ulceration burden have been extremely cost-effective in developing and developed countries. The challenge, principally in the less well-resourced health care systems, is implementing effective foot care realizing the potential health gains and cost-reductions ${ }^{(8)}$.

The management of diabetic foot has high cost globally, basically, the DFU management standard practices include wound off-loading, surgical debridement, infection, glycemic control, and dressings to facilitate a moist wound environment and exudation of control. These practices' best coordination is achieved via a multidisciplinary foot wound clinic ${ }^{(3)}$.

The wound dressings perform a crucial role in the cutaneous wounds' management due to their ability to protect wounds and promote dermal and epidermal tissue regeneration. Therefore, wound care has been a dramatic increase in the demand for better and suitable dressing because of the increase in the number of people are suffering from venous or arterial insufficiency, burns, trauma, chronic pressure, surgery, and diabetic ulcers. In general, an ideal dressing must-have property of mechanical robustness, non-toxicity, biocompatibility, and the appropriate permeability for exchanging water and gas (9-11).

Lim et al, (2017) recognized that the latest stage of foot ulcer complication had been associated with significant morbidity and an overall reduction in life quality. It has been estimated that greater than two-thirds (84\%) of the lower limb's amputations (non-traumatic) are preceded by an ulcer, a pivotal event opening the early intervention windows (12). Nevertheless, the core peripheral vascular disease's complicated factor makes most diabetic foot ulcers asymptomatic throughout the early stages. Whereas, in the more advanced stages, the tissue loss evidence becomes more apparent, most commonly occurring in the 
chronic non-healing foot ulcers form ${ }^{(13)}$.

initially, the wound-healing process for the diabetic foot encompasses the multifaceted biological mechanism activated by the injury, involves four crucial steps: (1) hemostasis, (2) inflammation, (3) proliferation, and (4) tissue remodeling. Accordingly, in all these processes, the occurrence of numerous biophysical and cellular event happens, like the differentiation of monocytes to macrophage, infiltration and lymphocytes and neutrophil, in the inflammation stage; or increased angiogenesis and reepithelialization by the migration of fibroblast, in the phase of proliferation (14).

Practically, an application of wound dressing can achieve the promotion of wound healing and prevention of bacterial infection. Many studies have reported the effectiveness of applying the silver-based dressings against pathogenic bacteria (15-19). Remarkably, the illustration of the silver colloid-based dressing has been proved an improvement in the dietetic ulcer wound healing process and minimizing the size of the ulcer, in considering the cost-effectiveness and reduce the burden in the health care system (19). The dressing preparation by the incorporation of an elevated Silver Nanoparticles (AgNPs) concentration contains sliver atoms that are discharged slowly positivesliver cations $(\mathrm{Ag}+)$, which provide a strong antibacterial activity against Staphylococcus aureus and Escherichia coli which causing destroy of the bacterial cell, whereas the integration of the Nanotechnology increasing the surface area with the production of smaller silver particles safely by limiting the toxicity of silver ions and promote the antiinflammatory activity, ultimately achieve the highest level of wound healing with lower toxicity (20-24).

The present study aimed to evaluate the effectiveness of using hydrogel/nano silver-based dressing vs. traditional dressing on diabetic foot wound healing.

\section{METHODS}

Study Design: The randomized controlled study was designed to compare the effectiveness of using hydrogel/nano silver-based dressing vs. traditional dressing on diabetic foot wound healing.

Participants and sampling: Sixty patients with type-2 diabetes hospitalized for diabetic foot wound treatment were recruited as a study sample. The study was done in selected Surgical departments at Kasr Al-Aini university hospital from January to April 2019.

Patients were assigned randomly into two groups by lot system. Group-I patients were subjected to hydrogellnano silver wound dressing, while group-II patients were subjected to a traditional wound dressing routine.

According to Kasr Al-Aini's medical protocol about anti-diabetic therapy, other needed medicine, and nutrition control, both groups of the current study were managed. The wound's physiological parameters, discharge color, odor, pain, wound size, and depth was assessed before dressing change.

Data Collection: The patients of group-I wounds were cleaned using normal saline; then, the wounds were closed firmly with hyderogel $\backslash$ nanosilver dressing. The dressing was changed every two days according to dressing manufacture instruction. When the dressing was opened after three days, the wound was assessed for granulation tissue, wound, and discharge size.

The patients of group II wounds were dresses using a traditional method, cleaned by normal saline, use of betadine antiseptic; then they were secured with clean linen gauze. The dressing was changed once daily. The wound healing process of the two groups was then assessed for three consecutive weeks.

Ethical Considerations: Formal letters were issued from the investigators to the Medical Director of Surgical departments at Kasr Al-Aini university hospital of the study settings and approval was obtained on the request so it signed and stamped from the hospital and from the Ethical Committee of the university (ClinicalTrials.gov Identifier: NCT04834245). The researcher met the patients and explained the aim and the process of the study. Patients' written consent was obtained. Complete confidentiality of any obtained information was ensured. The researcher has also assured the administration that the conduction of the study will not affect the work in the study settings. The results of the study will be provided to the hospitals' authorities to maximize their benefits.

Data analysis was carried out using the Statistical Package for Social Sciences (SPSS), version 20. All data entries were checked for accuracy against the original raw data of each patient by the investigator. The following statistical tests were used: (a) Frequency and percentage distribution for all variables, (b) Mean and standard deviation to describe the central tendency (c) t-test.

\section{RESULTS}

Total 60 patients divided into two groups, 30 patients who received traditional dressing were labeled as (traditional dressing) whereas the second group labeled as (Hydrogellnanosilver dressing).

The demographic characteristics of patients in the study sample are described in table (1), Patients' age ranged between 35 and 55 years with a mean \pm SD $46 \pm$ 2.05 years. Sex distribution was almost equal, with slightly more male $(55.0 \%)$. Half of them were working in a light active job and more than half of them had diabetes for 3-5 years $(55 \%)$.

Table (1): Distribution of the Patient's demographic Data:

\begin{tabular}{|l|l|l|}
\hline Item & Frequency $(\mathrm{N})$ & $\%(\mathrm{n}=60)$ \\
\hline Sex & 33 & \multicolumn{1}{|l|}{} \\
\hline Male & 33 & 55 \\
\hline Female & 27 & 45 \\
\hline Age range (years) & \multicolumn{1}{|l|}{} \\
\hline $35<45$ & 15 & 25 \\
\hline $45<55$ & 27 & 45 \\
\hline $55<65$ & 18 & 30 \\
\hline SD $\pm X$ & $46 \pm 2.05$ & 18.3 \\
\hline Type of job & \multicolumn{2}{|l|}{} \\
\hline Not active & 11 & 50 \\
\hline Light active job & 30 & 16.7 \\
\hline Moderate active job & 10 & 15 \\
\hline Active job & 9 & \\
\hline Duration of diabetes & \multicolumn{2}{|l|}{} \\
\hline \multicolumn{1}{|c|}{3 years } & 27 & 45 \\
\hline 3 -5 years & 33 & 55 \\
\hline
\end{tabular}

Figure (1) illustrates the diabetic foot wound's mean 
size from zero week to third week as in the traditional wound dressing group (5746.2, 3432, 1762.7, 827.9 respectively) was higher than the hydrogel/nanosilverbased dressing group (2582.8, 1661.9, 1157, 573.4 Respectively).

Figure 1: Mean area of the ulcer in each group (sq $\mathrm{mm}$ ):

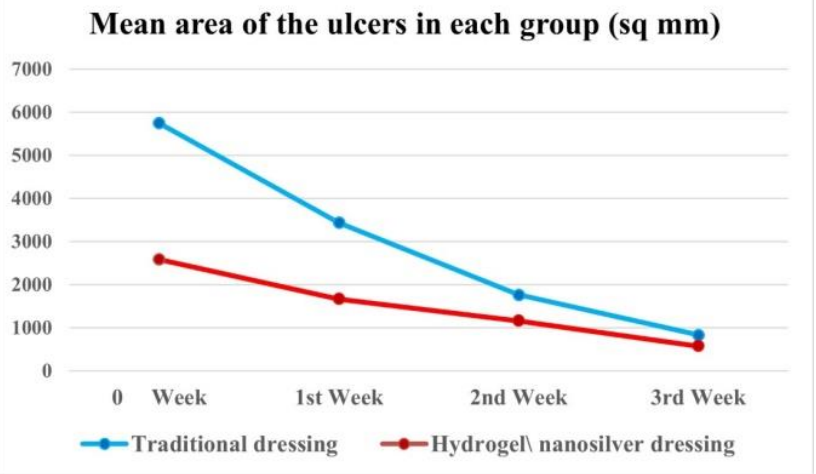

The rate of reduction of ulcer as a percentage of its initial area describes in Figure (2) and the percentages show a faster healing rate of ulcers in the hydrogel/nanosilver-based dressing group (100, 61.855, $35.84,15.11$ Respectively) from zero week to third week.
Figure 2: Rate of Reduction of ulcer as a percentage of its initial area:

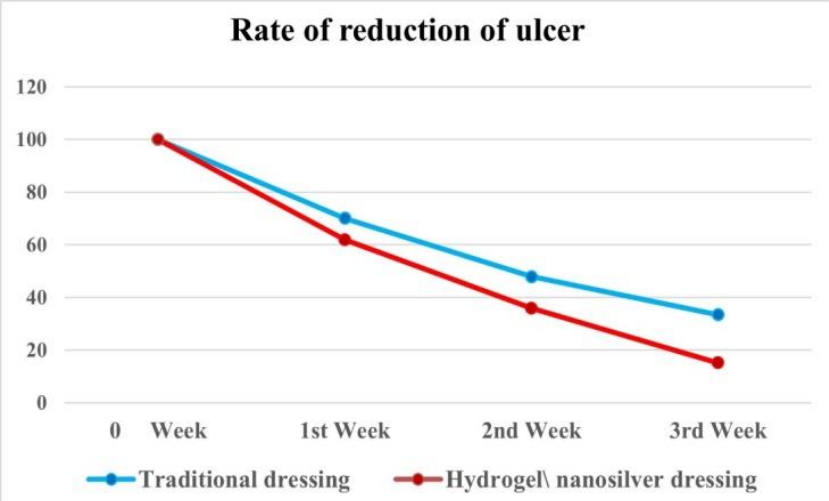

The use of hydrogellnanosilver wound dressing compared to the traditional dressing showed a more significant reduction in the area when the initial wound area is compared to the subsequent area measurements expressed as the initial percentage. However, the values were not statistically significant. However, healing and wound area reduction did occur faster in the hydrogel Inano silver wound dressing group (Table 2).

Table (2): Hydrogellnanosilver wound dressing vs. Traditional dressing (sq mm)

\begin{tabular}{|l|l|l|l|l|}
\hline Type of dressing & $\begin{array}{l}\text { Number of } \\
\text { Patients }\end{array}$ & $1^{\text {st }}$ week & $2^{\text {nd }}$ week & $3^{\text {rd }}$ week \\
\hline Hydrogellnanosilver wound dressing & 30 & $61.85 \pm 11.86$ & $35.84 \pm 8.35$ & $15.11 \pm 7.89$ \\
\hline Traditional dressing & 30 & $62.16 \pm 9.25$ & $37.70 \pm 12.50$ & $21.65 \pm 8.4$ \\
\hline P-value & 0.458 & 0.822 & 0.712 \\
\hline
\end{tabular}

\section{DISCUSSION}

Management of the foot ulcer is considering a great challenge, the healing of wounds depends on multifactor that affect the healing process, alongside the huge burden on patients, the health care system, and society ${ }^{(25)}$.

The present randomized control study was carried out on 60 patients, randomly selected, their personal characteristics are similar to those of patients with diabetes in Egypt as reported by Al-Ghazaly et al, 2015 and AlNakeeb et al, 2021(26,27).

Initially, the wound starts unintentionally, therefore wounds are colonized with microorganisms causing a serious diabetic foot infection once the wound becomes infected with purulent exudate and inflammation may lead to DFU and ends by leg amputation (7). Practically, selecting a suitable type of advanced antimicrobial dressing to play a key part of DFU treatment as Honey-impregnated dressings, lodine-Impregnated dressing, and silverimpregnated dressing (28). Recently, the integration of silver with nanoparticles displays a significant novel and distinguish of physical, chemical, and biological characteristics, meanwhile, the magic nanoscale size provides a unique tool in tissue repair and overall wound management ${ }^{29,30)}$.

Stoica et al, 2020, reported that the traditional conventional wound dressing may provide wound dehydration, attached directly to the wound cause pain and restlessness ${ }^{(30)}$.

In congruence with the present study findings (Suhas \& Manvi, 2018) have investigated the effectiveness of applying the Nano-silver on wound healing and found the wound healing improves, provides antimicrobial activity, and promotes size ulcer reduction ${ }^{(29)}$.

After the implementation of the hydrogel/nano silverbased dressing on patient's with DFU of the present study, the findings illustrated that use of hydrogellnano silver wound dressing compared to the traditional dressing showed a more significant reduction in the area when the initial wound area is compared to the subsequent area measurements expressed as the initial percentage. The wound healing is in agreement with Essa et al 2021, who have recently compared silver Nanoparticles dressing and conventional dressing in diabetic foot healing, and their findings proved that the healing rate was statistically significant and the healing rate per week and the complete recovery was achieved with SliverSTAT group, around onefifth of the study sample by six weeks where the rest of the patients were healed from week eight to week twelve, whereas the conventional group the wound healing started from week eight to week twelve ${ }^{(31)}$.

In the same line Sharma et al, 2017 have conducted a randomized clinical trial to identify the effect of the silver colloid dressing over conventional dressings and reported significant wound healing in size in silver colloid-based 
dressing compared to conventional dressing in DFU ( $p$ $<0.05)^{(19)}$. In disagreement with the present study finding, a study have done by Rayman et al, 2005 and reported that only four DFU were healed completely treated by silver dressing (56\%) whereas six DFU were not healed ${ }^{(32)}$.

\section{CONCLUSION}

The overall cost of dressing was lesser in the hydrogell nanosilver dressing group with decreasing hospital stay. There were no side effects in the hydrogell nanosilver dressing group can be considered a superior option in managing chronic wounds. Hydrogel\ nanosilver dressing increases wound healing by increasing the rate of reepithelialization and wound contraction as observed by the faster reduction in the wound area. More extensive and indepth studies are required to investigate this effect of the therapy. Hydrogel $\backslash$ nanosilver dressing is a safe and effective adjuvant modality of treatment for diabetic foot ulcers.

Limitations of the study: The sample size is considerably small. A randomized control study with a much larger population may further substantiate the findings or reveal variations.

The patients' cost burden can be influenced by many factors other than dressing materials alone. The hyderogel nanosilver dressing in this study is considered a high cost for some patients. However, the dressings' overall cost was lesser as it was done only once in 3 days, whereas in other groups, the dressing was done daily.

The assessment of results such as graft uptake, recurrence of the ulcer, residual raw area, and the pain was not included in the study, which might have given a much better analysis of the efficacy of hyderogel $\backslash$ nanosilver dressing as compared to the traditional method of dressing.

Funding: No funding sources

Conflict of interest: None declared

\section{REFERENCE}

1. American Diabetes Association (ADA). Standards of medical care in diabetes-2016. Diabetes Care 2016a;39(Suppl 1), S1-S2.

2. Ezhilarasu $H$, Vishalli D, Dheen $T$, Bay B and Srinivasan D, 2.Nanoparticle-Based Therapeutic Approach for Diabetic Wound Healing. Nanomaterials 2020; 10, 1234; doi:10.3390/nano10061234.

3. Everett, E., \& Mathioudakis, N. Update on management of diabetic foot ulcers. Annals of the New York Academy of Sciences 2018; 1411(1), 153-165. https://doi.org/10.1111/nyas.13569.

4. Amogne W, Reja A, Amare A. Diabetic foot disease in Ethiopian patients: A hospital-based study. Ethiiop J Health Dev 2011;25(1).

5. El-Nahas M, Gawish $\mathrm{H}$, Tarshoby $M$, State $\mathrm{O}$. The prevalence of risk factors for foot ulceration in Egyptian diabetic patients. Practical Diabetes International 2008; 25(9):362-366.

6. Rizk, M.N \& Ameen, A.I. (2013). Comorbidities associated with Egyptian diabetic foot disease subtypes. The Egyptian Journal of Internal Medicine 2013, (25)154-158.

7. Lipsky B, Berendt A, Cornia P, Pile J, Peters E, Armstrong D, Deery G, Embil J, Joseph W, Karchmer A, Pinzur M,11 and Senneville E. 2012 Infectious Diseases Society of America Clinical Practice Guideline for the Diagnosis and Treatment of Diabetic Foot Infections. IDSA Guideline for Diabetic Foot Infections, CID 2012, 54 1679-1684.
8. Cavanagh, P., Attinger, C., Abbas, Z., Bal, A., Rojas, N. \& $\mathrm{Xu}$, Z.R. Cost of treating diabetic foot ulcers in five different countries. Diabetes Metab J 2012; 28 (1):107-11.

9. Liu, M., Liu, T., Chen, X., Yang, J., Deng, J., He, W., Zhang, X., Lei, Q., Hu, X., Luo, G., \& Wu, J. Nano-silverincorporated biomimetic polydopamine coating on a thermoplastic polyurethane porous nanocomposite as an efficient antibacterial wound dressing. Journal of nanobiotechnology 2018; 16(1), 89.

10. O'Meara SM, Cullum NA, Majid M, Sheldon TA. Systematic review of antimicrobial agents used for chronic wounds. $\mathrm{Br} \mathrm{J}$ Surg 2001;88(1):4-21.

11. O'Meara S, Al-Kurdi D, Ologun Y, Ovington LG, Martyn-St James M, Richardson R. Antibiotics and antiseptics for venous leg ulcers. Cochrane Database Syst Rev. 2013;12.

12. Lim, J. Z., Ng, N. S., \& Thomas, C. Prevention and treatment of diabetic foot ulcers. Journal of the Royal Society of Medicine 2017; 110(3), 104-109.

13. Tuttolomondo A, Maida C, Pinto A. Diabetic foot syndrome: Immune-inflammatory features as possible cardiovascular markers in diabetes. World J Orthop 2015;8, 6(1): 62-76.

14. Gomes, A., Teixeira, C., Ferraz, R., Prudêncio, C., \& Gomes, P. Wound-Healing Peptides for Treatment of Chronic Diabetic Foot Ulcers and Other Infected Skin Injuries. Molecules 2017; 22(10), 1743.

15. Brett DW. A discussion of silver as an antimicrobial agent: alleviating the confusion. Ostomy Wound Manage 2006; 52:1,34-41.

16. Percival SL, Bowler PG, Russell D. Bacterial resistance to silver in wound care. J Hosp Infect. 2005; 60:1,1-7.

17. Lansdown AB. Silver absorption and antibacterial efficacy of silver dressings. J Wound Care. 2005; 14:4,155-160.

18. Thomas $S$, McCubbin $P$. An in vitro analysis of the antimicrobial properties of 10 silver-containing dressings. J Wound Care. 2003; 12:8,101-7.

19. Sharma R, Gupta N, Kumar V, Pal S, Sharma R, Kaundal V, Sharma V. Silver colloid dressings score over conventional dressings in diabetic foot ulcer: a randomized clinical trial. International Surgery Journal 2017;4(8):2627-2631.

20. Liu, M., Luo, G., Wang, Y., Xu, R., Wang, Y., He, W., Tan, J., Xing, M., \& Wu, J. Nano-silver-decorated microfibrous eggshell membrane: processing, cytotoxicity assessment and optimization, antibacterial activity and wound healing. Scientific reports 2017; 7(1), 436.

21. Cahn $A$ and Kleinman $Y$, "A novel approach to the treatment of diabetic foot abscesses-a case series," Journal of Wound Care 2014; 23, 8: 394-399.

22. Lipsky B, Berendt A, Cornia P et al., "2012 infectious diseases society of America clinical practice guideline for the diagnosis and treatment of diabetic foot infections," Clinical Infectious Diseases 2012; (54)12: e132-e173.

23. White W, "Sharp wound debridement in the management of recalcitrant, locally infected chronic venous leg ulcers: a narrative review," Wound Practice and Research. 2011; 19, 4.

24. Bradshaw C, "An in vitro comparison of the antimicrobial activity of honey, iodine and silver wound dressings," Bioscience Horizons 2011; 4, 1:61-70.

25. Schaper NC, Van Netten JJ, Apelqvist J, Lipsky BA, Bakker $\mathrm{K}$, Internationa Working Group on the Diabetic Foot. Prevention and management of foot problems in diabetes: a summary guidance for daily practice 2015 , based on the IWGDF guidance documents. Diabetes Metab Res Rev 2016; 32,1:7-15.

26. Al-Ghazaly G, Al-Ahwal L, Zagloul K. Risk factors of diabetic foot ulcer in patients attending diabetic foot outpatient clinic at Tanta University Hospital in Egypt. The Journal of Diabetic Foot Complications, 2015; 7, 2, 3:42-48.

27. Al-Nakeeb AM, State OI, Tarshoby MM, Kyrillos FA. Risk factors for foot ulceration among adult patients with diabetes 
on chronic hemodialysis in Dakahlia Governorate, Egypt. J Egypt Soc Nephrol Transplant 2021;21:18-24.

28. Dumville JC, O'Meara S, Deshpande S, Speak K. Hydrogel dressings for healing diabetic foot ulcers. Cochrane Database of Systematic Reviews 2013, 7. CD009101.

29. Suhas K, Manvi N. Efficacy of nano silver dressings over conventional dressings in chronic wounds. Int Surg J. 2018;5(12):3995-3999.

30. Stoica A, Chircov C, Grumezescu A. Nanomaterials for Wound Dressings: An Up-to-Date Overview. Molecules
2020, 25, 2699.

31. Essa M ,Ahmad K, Zayed M, Ibrahim S. Comparative Study Between Silver Nanoparticles Dressing (SilvrSTAT Gel) and Conventional Dressing in Diabetic Foot Ulcer Healing: A Prospective Randomized Study. Int J Low Extrem Wounds, 2021;9;1534734620988217.

32. Rayman G, Rayman A, Baker NR, Jurgeviciene N, Dargis V, Sulcaite $R$, et al. Sustained silver-releasing dressing in the treatment of diabetic foot ulcers. Br J Nur. 2005;14(2). 